ARTICLE

https://doi.org/10.1038/s41467-020-17661-z

\title{
The suboptimality of perceptual decision making with multiple alternatives
}

\author{
Jiwon Yeon (D) ${ }^{1 凶} \&$ Dobromir Rahnev (D) ${ }^{1 凶}$
}

It is becoming widely appreciated that human perceptual decision making is suboptimal but the nature and origins of this suboptimality remain poorly understood. Most past research has employed tasks with two stimulus categories, but such designs cannot fully capture the limitations inherent in naturalistic perceptual decisions where choices are rarely between only two alternatives. We conduct four experiments with tasks involving multiple alternatives and use computational modeling to determine the decision-level representation on which the perceptual decisions are based. The results from all four experiments point to the existence of robust suboptimality such that most of the information in the sensory representation is lost during the transformation to a decision-level representation. These results reveal severe limits in the quality of decision-level representations for multiple alternatives and have strong implications about perceptual decision making in naturalistic settings.

\footnotetext{
${ }^{1}$ School of Psychology, Georgia Institute of Technology, Atlanta, GA, USA. ${ }^{凶}$ email: j.yeon@gatech.edu; rahnev@psych.gatech.edu
} 
$\mathrm{P}$ erception has been conceptualized as a process of inference for over a century and a half ${ }^{1}$. According to this view, the outside world is encoded in a pattern of neural firing and the brain needs to decide what these patterns signify. Hundreds of papers have revealed that this inference process is suboptimal in a number of different ways ${ }^{2}$. However, these papers have almost exclusively employed tasks with only two stimulus categories (though notable exceptions exist ${ }^{3,4}$ ). Experimental designs where decisions are always between two alternatives cannot fully capture the processes inherent in naturalistic perceptual decisions where stimuli can belong to many different categories (e.g., which of all possible local species does a particular tree belong to). Therefore, fully understanding the mechanisms and limitations of perceptual decision making requires that we characterize the process of making decisions with multiple alternatives.

One critical difference between perceptual decisions with two versus multiple alternatives is the richness of the sensory information that the decisions are based on. Decisions with two alternatives can be based on the evidence for each of the two categories, or even just the difference between these two pieces of evidence ${ }^{5}$. For example, traditional theories such as signal detection theory ${ }^{6}$ and the drift diffusion $\operatorname{model}^{7}$ postulate that two-choice tasks are performed by first summarizing the evidence down to a single number-the location on the evidence axis in signal detection theory and the identity of the boundary that is crossed in drift diffusion-that is subsequently used for decision making. Thus, the sensory information relevant for the decision in such tasks is relatively simple and could potentially be represented in decision-making circuits without substantial loss of information. However, the relevant sensory information in multi-alternative decisions is more complex because it contains the evidence for each of the multiple alternatives available. Further, this richer sensory information can no longer be summarized in a simple form in decision-making circuits without a substantial loss of information (Fig. 1). However, it is currently unknown whether decision-making circuits can represent the rich information from sensory circuits in the context of multi-alternative decisions or whether the decisionmaking circuits only represent a crude summary of the sensory representation.

To uncover the decision-level representation of decisions with multiple alternatives, we use discrete stimulus categories in three experiments and stimuli that give rise to a trimodal sensory distribution in a fourth experiment. All experiments feature a condition where subjects pick the dominant stimulus among all of the possible stimulus categories (four different colors in Experiment 1, six different symbols in Experiments 2 and 3, and three different stimulus direction in Experiment 4). Based on these responses, we estimate the parameters of a model describing subjects' internal distribution of sensory responses (i.e., the activity levels for each stimulus category). We then include conditions where subjects are told to pick between only two alternatives after the offset of the stimulus (Experiments 1, 2, and 4) or to make a second choice if the first one is incorrect (Experiment 3). These conditions allow us to compare different models of how the sensory representation is transformed into a decision-level representation. To anticipate, we find robust evidence for suboptimality in that decisions in our experiments are based on a summary of the sensory representation thus incurring substantial information loss. These results indicate that perceptual decision-making circuits may not have access to the full sensory representation in the context of multiple alternatives and that significant amount of simplification is likely to occur before sensory information is used for deliberate decisions.

\section{Results}

Experiment 1. Subjects saw a briefly presented stimulus consisting of 49 colored circles arranged in a $7 \times 7$ square (Fig. 2). Each circle was colored in blue, red, green, or white. On each trial, one color was randomly chosen to be dominant (i.e., more frequently presented than the other colors) and 16 circles were painted in that color, whereas the remaining three colors were nondominant and 11 circles were painted in each of those colors. The task was to indicate the dominant color. The experiment featured two different conditions. In the four-alternative condition, subjects picked the dominant color among the four possible colors. In the two-alternative condition, after the offset of the stimulus, subjects were asked to choose between the dominant and one randomly chosen nondominant color. In both conditions, the response screen was displayed with 0 -ms delay thus minimizing short-term memory demands. Note that subjects' task was always the same (to correctly identify the dominant color).

Based on the responses in the four-alternative condition, we estimated the parameters of the sensory distribution representing the activity level for each color. We then considered the predictions for the two-alternative condition of two different models: (1) a population model, according to which perceptual decisions are based on the whole distribution of activities over the four colors, and (2) a summary model, according to which perceptual decisions are based on a summary of the whole distribution. There are a number of ways to create a summary of the whole distribution. However, in the context of this task, the only relevant information is the order of the activation levels from highest to lowest (this order determines how a subject would pick different colors as the dominant color in the two-alternative condition). Other information, such as average activity level, is irrelevant to the task here. Therefore, we first considered an extreme summary model that consists of the activity level for the one color with highest level of activity. Other summary models, in which decision-making circuits have access to the activity levels of the $n>1$ colors with highest activity levels, are examined later.

The population and summary models could be easily compared because they make different predictions about performance in the two-alternative task (for a mathematical derivation, see Supplementary Methods). Indeed, the models make the same prediction when the dominant color gives rise to the highest activity level (Fig. 3a) and when the alternative option given to the subject happens to have the highest activity (Fig. 3b), but diverge when the highest activity is associated with a color that is not among the two options with the population model predicting a higher performance level (Fig. 3c).

The difference between the two models could be seen in the actual model predictions. Indeed, based on the performance in the four-alternative condition (average accuracy $=69.2 \%$, chance level $=25 \%$ ), the population and summary models predicted an average accuracy of $84.2 \%$ and $79.7 \%$ in the two-alternative condition, respectively. Compared to the actual subject performance (average accuracy $=78 \%$ ), the population model overestimated the accuracy in the two-alternative conditions for 29 of the 32 subjects (average difference $=6.21 \% ; t(31)=8.19, p=$ $\left.3.02 \times 10^{-9}, 95 \% \mathrm{CI}=[4.7 \%, 7.8 \%]\right)$. Surprisingly, the summary model also overestimated the accuracy in the two-alternative condition but the misprediction was much smaller (average difference $=1.72 \% ; t(31)=2.35, p=0.025,95 \% \quad \mathrm{CI}=[0.2 \%$, $3.2 \%]$ ) (Fig. 4a). Indeed, the absolute error of the predictions of the population model was significantly larger than for the summary model (average difference $=2.93 \% ; t(31)=5.65, p=$ $\left.3.34 \times 10^{-6}, 95 \% \mathrm{CI}=[1.9 \%, 4 \%]\right)$. Overall, the summary model predicted the accuracy in the two-alternative condition better than the population model for 26 of the 32 subjects (Fig. $4 \mathrm{~b}$ ). 
a

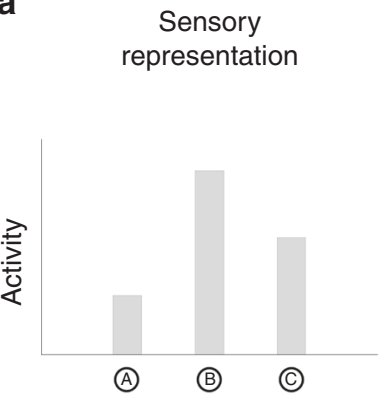

(C)

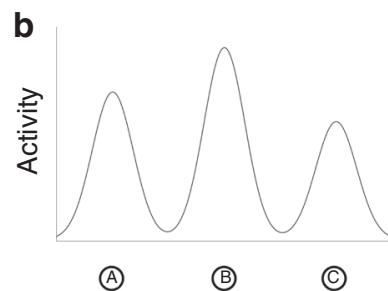

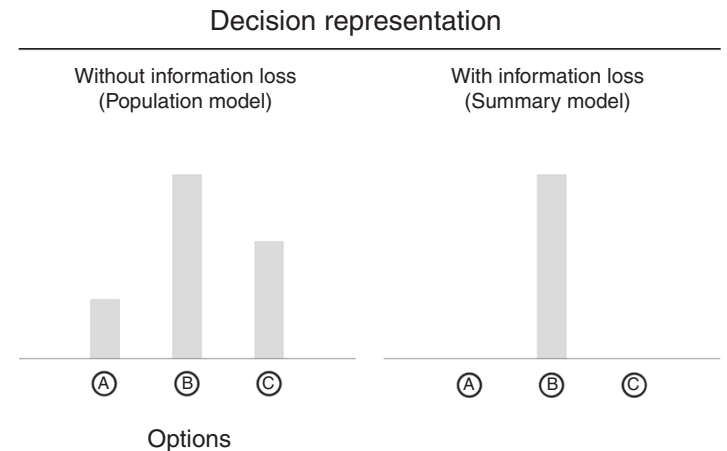

Options

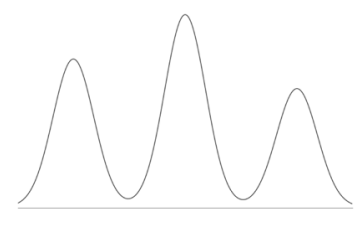

(A)
(B)

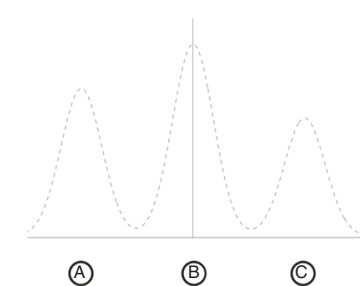

Orientation

Fig. 1 Sensory and decision-level representations for multiple alternatives. a Illustration of decision making with multiple discrete alternatives. In cases where a subject has to choose between multiple discrete alternatives (e.g., options A, B, and C), a stimulus can be assumed to give rise to a sensory representation that consists of different amount of sensory activity for each alternative (left panel). A decision-level representation without information loss would consist of a copy for the sensory representation (middle panel). We refer to this possibility as a population model of decision-level representation. On the other hand, the decision-level representation may consist of only a summary of the sensory representation thus incurring information loss. One possible summary representation consists of passing only the highest activity onto decision-making circuits (right panel). We refer to this type of representation as a summary model of decision-level representation. This summary representation involves information loss that will become apparent if subjects have to choose between the other alternatives (e.g., alternatives A and C). b Decision making with a continuous but multimodal sensory representation. Similar to having multiple discrete alternatives, decisions can involve judging a continuous feature (e.g., orientation) but in the context of a multimodal (e.g., a trimodal) underlying sensory representation (left panel). The decision-level representation can again consist of either a copy for the sensory representation (middle panel) or a summary of this sensory representation (right panel).

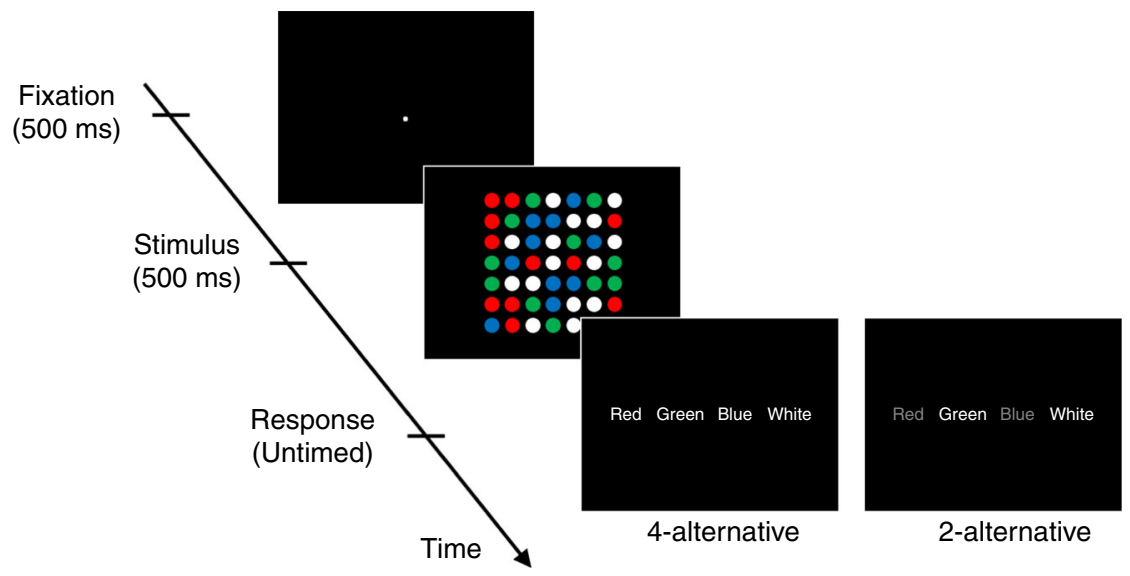

Fig. 2 Task for Experiment 1. Each trial consisted of a fixation period ( $500 \mathrm{~ms}$ ), stimulus presentation ( 500 ms), and untimed response period. The stimulus comprised of 49 circles each colored in one of four different colors (red, green, blue, and white). One of the colors (white in this example) was presented more frequently ( 16 circles; dominant color) than the other colors ( 11 circles each; nondominant colors). The task was to indicate the dominant color. Two conditions were presented in different blocks. In the four-alternative condition, subjects chose between all four colors. In a separate twoalternative condition, on each trial subjects were given a choice between the dominant and one randomly chosen nondominant color.

We further compared the models' fits to the whole distribution of responses. We found that the Akaike Information Criterion (AIC) favored the summary model by an average of 24.30 points (Fig. 4c), which corresponds to the summary model being $1.89 \times$ $10^{5}$ times more likely than the population model for the average subject. Across the whole group of 32 subjects, the total AIC difference was thus 777.63 points, corresponding to the summary model being $7.26 \times 10^{168}$ times more likely in the group. Note that since the population and summary models had the same number of parameters, the same results would be obtained regardless of the exact metric employed (e.g., the BIC differences would be exactly the same; see "Methods" for details).

Finally, we constructed and tested four additional models. The first two models postulated that decision-making 
a Highest activity in the dominant color

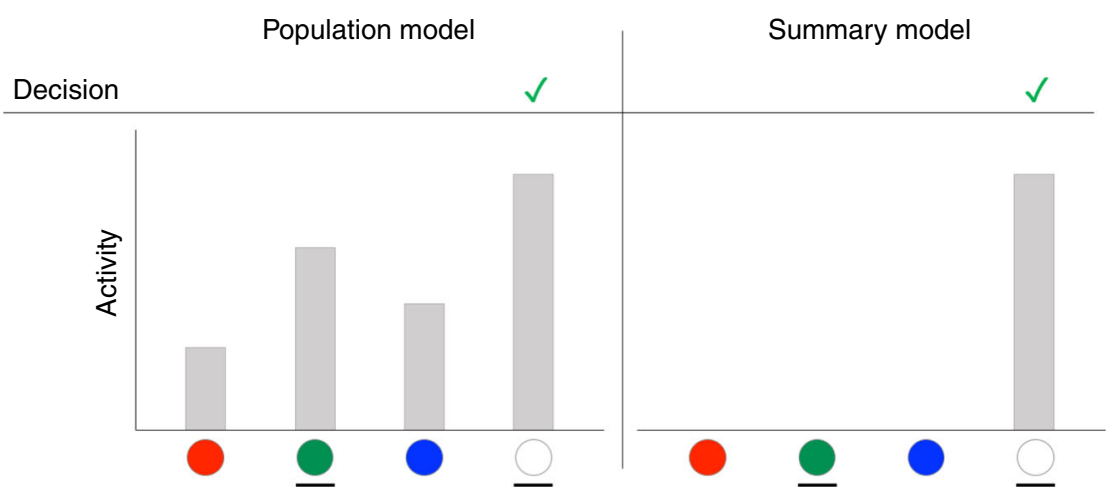

b Highest activity in the alternative color

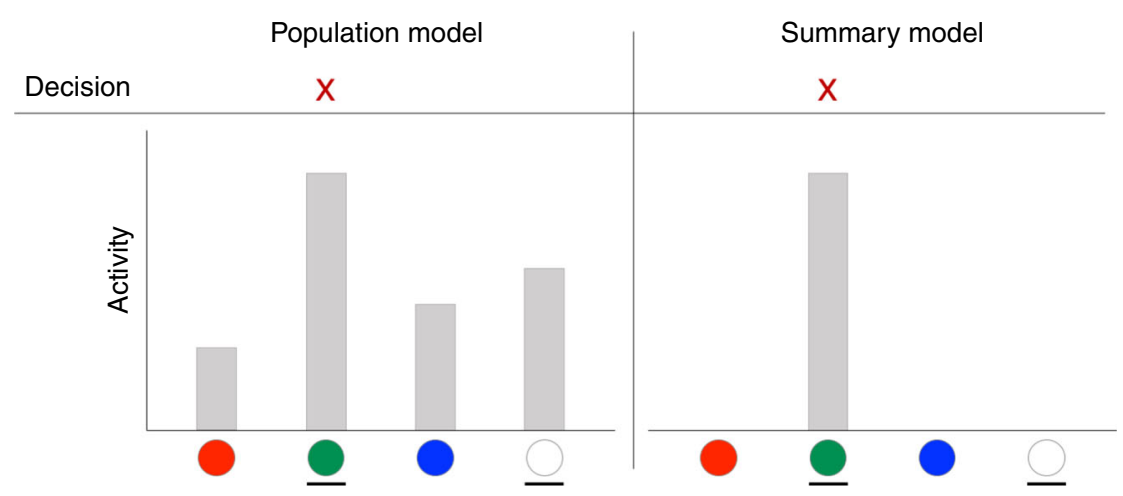

C Highest activity in a color that is not among the two alternatives

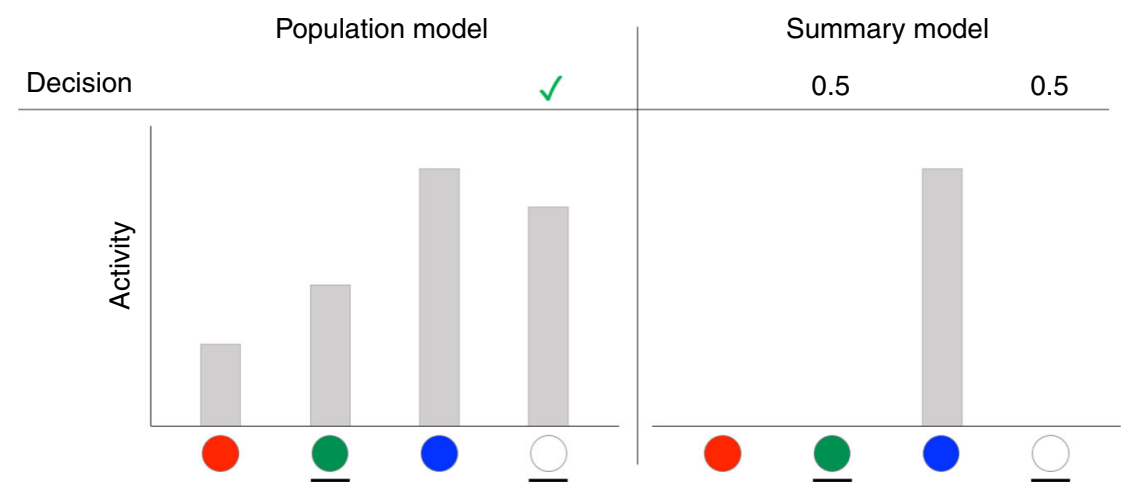

Fig. 3 Predictions of the two models for choices in the two-alternative condition. The population model (left panels) assumes that decision-making circuits have access to the activity levels associated with each of the four colors (four gray bars), whereas the summary model (right panels) assumes that decision-making circuits only have access to the highest activity level (single gray bar). In all examples, the dominant circle is white, and subjects are given a choice between white and green. a When the highest activity happens to be at the dominant color, both models predict that the subject would correctly choose the dominant color. b When the highest activity happens to be at the alternative color, both models predict that the subject would incorrectly choose the alternative color. c The predictions of the two models diverge when the highest activity is associated with a color other than the two presented alternatives. In such cases, the activation for the dominant color is likely to be higher than for the alternative color, so according to the population model, subjects would ignore the color with the highest activity (blue color in the example here) and correctly pick the dominant color in the majority of the trials. However, according to the summary model, subjects have no information about the activation levels for the dominant and the alternative colors and would thus correctly pick the dominant color on only $50 \%$ of such trials.

circuits have access to the two or three highest activations of the sensory distribution (two-highest and three-highest models, respectively). These models could thus be seen as intermediate options between the summary and population models. Two other models postulated that subjects choose either two or three stimulus categories to attend to and then make their decisions based on a full probability distribution over the activity levels of the attended categories (two-attention and three-attention models; see Supplementary Methods for details). We found that all of these models were outperformed by the summary model (Supplementary Notes and Supplementary Figs. 1, 6a-d). 
a

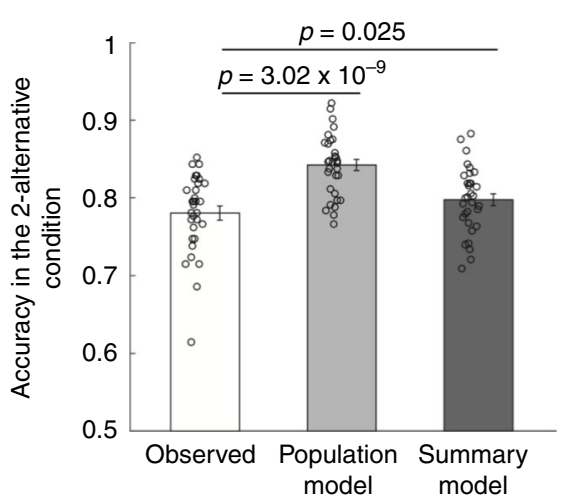

b

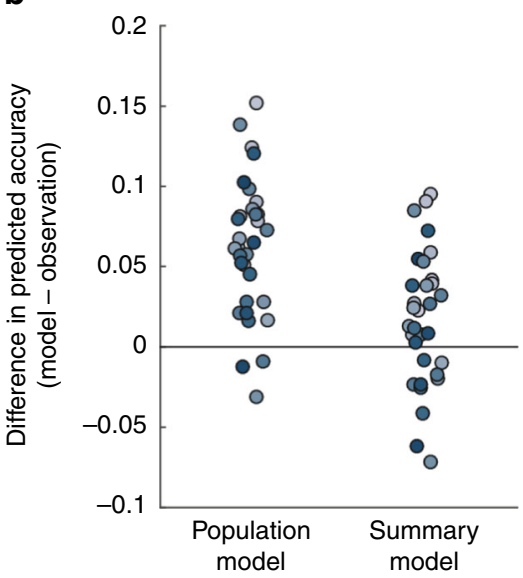

C

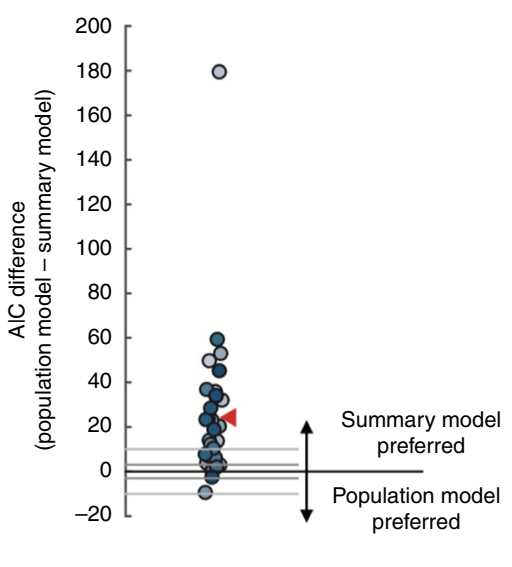

Fig. 4 Comparisons between the population and summary models in Experiment 1. a Mean accuracy in the two-alternative condition observed in the actual data (white bar), and predicted by the population (light gray bar) and summary (dark gray bar) models. The predictions for both models were derived based on the data in the four-alternative condition. All $p$ values are derived from two-sided paired $t$ tests. Error bars represent SEM, $n=32$. b Individual subjects' differences in the accuracy in the two-alternative condition between the two models and the observed data. c Difference in Akaike Information Criterion (AIC) between the population and the summary models. Positive AIC values indicate that the summary model provides a better fit to the data. Each dot represents one subject. The gray horizontal lines at \pm 3 and \pm 10 indicate common thresholds for suggestive and strong evidence for one model over another. The red triangle indicates the average AIC difference. The summary model provided a better fit than the population model for 30 of the 32 subjects.

Experiment 2. The results from Experiment 1 strongly suggest that within the context of our experiment, decision-making circuits do not represent the whole sensory distribution but only a summary of it. We sought to confirm and generalize these findings in two additional, preregistered experiments ([osf.io/dr89k/]). For Experiment 2, we made several modifications: (1) we changed the colored circles to symbols, (2) we raised the number of stimulus categories from four to six, and (3) we significantly increased the number of trials per subject in order to obtain stronger results at the individual-subject level. Specifically, we presented the six symbols (?, \#, \$, \%, +, and >) such that the dominant symbol was presented 14 times and each nondominant symbol was presented 7 times (Fig. 5a). The 49 total symbols were again arranged in a $7 \times 7$ grid. Each subject completed a total of 3000 trials that included equal number of trials of a six-alternative condition and a two-alternative condition that were equivalent to the four- and two-alternative conditions in Experiment 1.

Just as in Experiment 1, we computed the parameters of the sensory representation using the trials from the six-alternative condition (average accuracy $=50.5 \%$, chance level $=16.7 \%$ ) and used these parameters to compare the predictions of the population and summary models for the two-alternative condition. We found that the average accuracy in the two-alternative condition (71.6\%) was slightly underestimated by the summary model (predicted accuracy $=70.1 \% ; \quad t(9)=-2.76, p=0.022,95 \%$ CI: $[-2.7 \%$, $-0.3 \%]$ ) but was again significantly overestimated by the population model (predicted accuracy $=77.5 \%$; $t(9)=9.41, p=$ $5.92 \times 10^{-6}, 95 \%$ CI $[4.5 \%, 7.3 \%]$ ) (Fig. 5b). Individually, the summary model provided better prediction of the accuracy in the two-alternative condition for nine out of the ten subjects (Fig. 5c).

Further, we compared the population and summary models' fits to the whole distribution of responses. We found that the summary model was preferred by nine of our ten subjects and the difference in AIC values in all these nine subjects was larger than 25 points (Fig. $5 \mathrm{~d}$ ). The AIC values of the one subject for whom the population model was favored over the summary model differed by only 7.9 points. On average, the summary model had an AIC value that was 57.79 points lower than the population model corresponding to the summary model being $3.55 \times 10^{12}$ times more likely for the average subject. Across the whole group of ten subjects, the total AIC difference was thus 577.94 points, corresponding to the summary model being $3.14 \times 10^{125}$ times more likely in the group. Finally, we found that the additional four models were again outperformed by the summary model (Supplementary Notes and Supplementary Figs. 2, 6e-h).

Experiment 3. Taken together, Experiments 1 and 2 suggest that in the context of multi-alternative decisions, the system for deliberate decision making may not have access to the whole sensory representation. This conclusion is based on experiments that differed in the nature of the stimulus, the number of stimulus categories, and the amount of trials that subjects performed. Nevertheless, both Experiments 1 and 2 relied on the same design of comparing four- (or six-) and two-alternative conditions. Therefore, to further establish the generality of our results, in Experiment 3 we employed a different experimental design. We used the same stimulus as in Experiment 2 and presented all six alternatives on every trial, but additionally gave subjects the opportunity to provide a second answer on about $40 \%$ of error trials (Fig. 6a). Using the performance on the first answer, we compared the predictions of the population and summary models for the second answers.

The population model makes a clear prediction about the second answer-subjects should choose the stimulus category with the highest activation from among the remaining five options. The second answer will thus have relatively high accuracy because the presented stimulus category is likely to produce one of the highest activity levels (Supplementary Fig. 3a). On the other hand, the summary model only features information about the stimulus category with the highest activity. Once that stimulus category is chosen as the first answer, the model postulates that the subject does not have access to the activations associated with the other stimulus categories. Given this representation, subjects could adopt at least two different response strategies. One possible strategy is for the subject to make their second answer at random, which would result in chance level (20\%) performance. We call this the Summary \& Random Choice model (Supplementary Fig. 3b). However, 
a

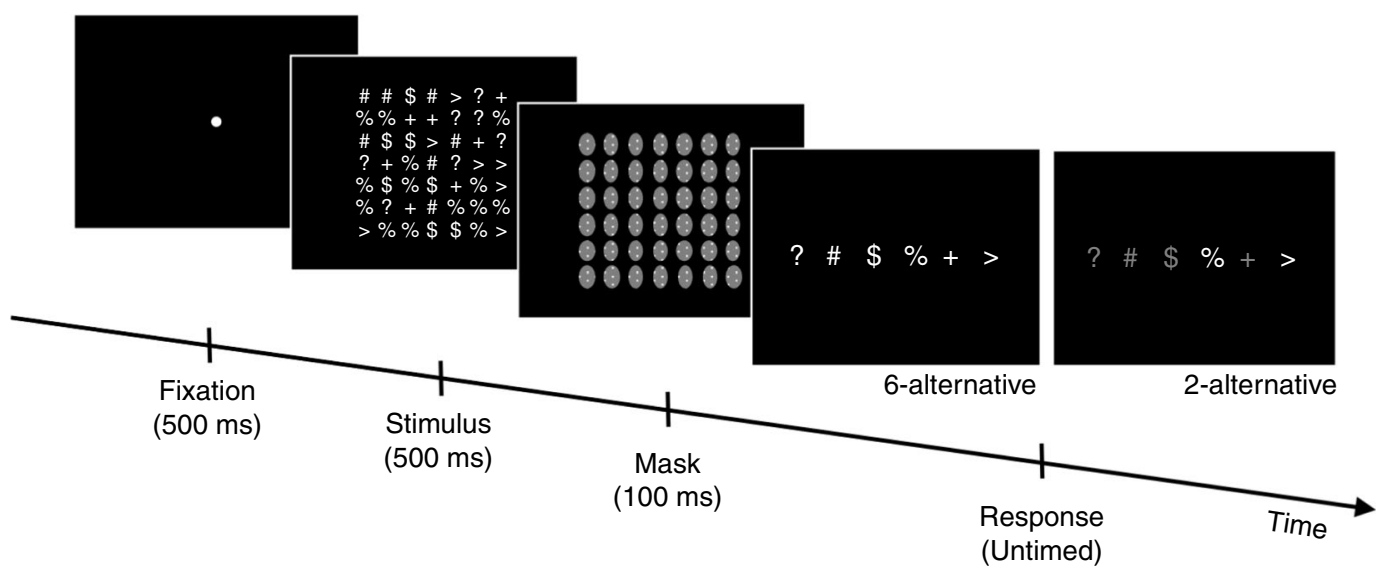

b

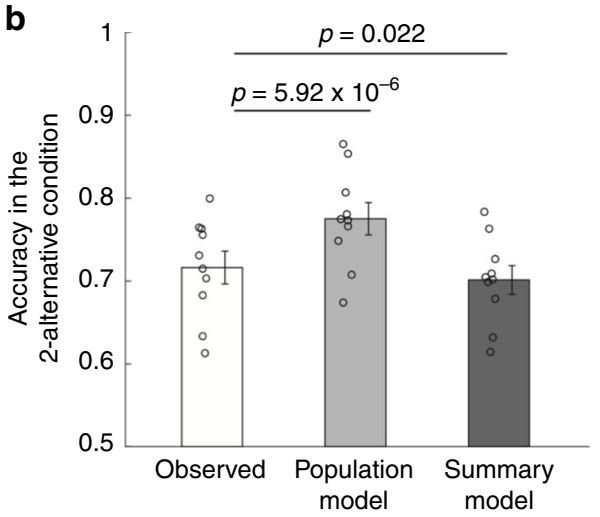

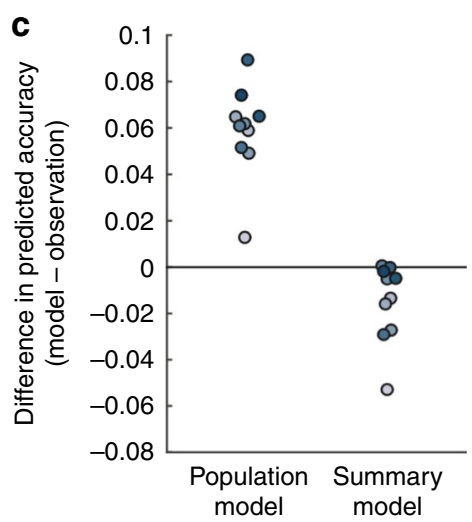

d

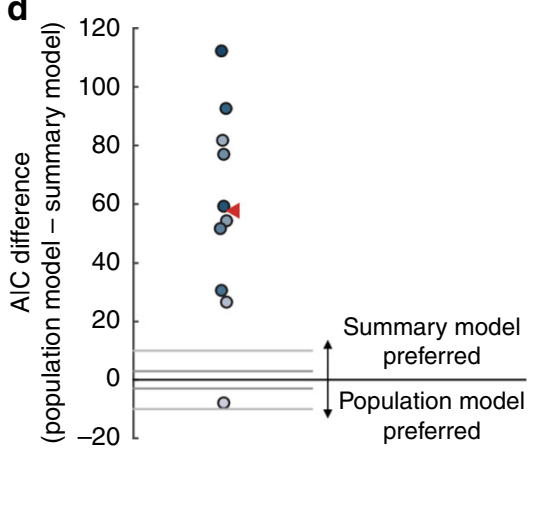

Fig. 5 Task and results for Experiment 2. a The task in Experiment 2 was similar to Experiment 1 except for using six different symbols (question mark, pound sign, dollar sign, percentage sign, plus sign, and greater-than sign) instead of four different colors. One of the symbols was presented more frequently (14 times, dominant symbol) than the others ( 7 times each, nondominant symbols) and the task was to indicate the dominant symbol. Two conditions were presented in different blocks: a six-alternative condition where subjects chose between all six symbols and a two-alternative condition where subjects were given a choice between the dominant and one randomly chosen nondominant symbol. $\mathbf{b}$ Mean accuracy in the two-alternative condition observed in the actual data (white bar) and predicted by the population (light gray bar) and summary (dark gray bar) models. The predictions for both models were derived based on the data in the six-alternative condition. All $p$ values are derived from two-sided paired $t$ tests. Error bars represent $\mathrm{SEM}, n=10$. $\mathbf{c}$ Individual subjects' differences in the accuracy of the two-alternative condition between the two models and the observed data. $\mathbf{d}$ AIC difference between the population and the summary models. Positive AIC values indicate that the summary model provides a better fit to the data. Each dot represents one subject. The red triangle indicates the average AIC difference. The summary model provided a better fit than the population model for nine out of ten subjects.

another possibility is for the subject to make the second answer strategically. One available strategy is for the subject to pick the stimulus category of a randomly recalled symbol from the $7 \times 7$ grid. Given that subjects inspected the stimuli for $500 \mathrm{~ms}$, they could easily remember one location with a symbol other than the one they picked for their first answer. We call this the Summary \& Strategic Choice model (Supplementary Fig. 3c). According to this model, the second answer will be correct on $\frac{14 \text { (\# locations of the dominant symbol) }}{42 \text { (total\# of remaining locations) }}=\frac{1}{3}$ or $33.3 \%$ of the time. Conversely, each of the four remaining incorrect categories will be chosen on $\frac{7 \text { (\# locations of each nondominant symbol) }}{42 \text { (total } \# \text { of remaining locations) }}=\frac{1}{6}$ or $16.7 \%$ of the time (Supplementary Fig. 4).

To adjudicate between these three models, we first examined subjects' accuracy on the first answer. Subjects responded correctly in their first answer on $50.7 \%$ of the trials (chance level $=16.7 \%$ ). Using this performance, we computed the parameters of the sensory representation as in Experiments 1 and 2 in order to generate the model predictions for the second answer. We found that task accuracy for the second answers was $29.6 \%$. This value was greatly overestimated by the population model, which predicted accuracy of $40.9 \%(t(9)=7.04, p=$ $\left.6.09 \times 10^{-5}, 95 \% \mathrm{CI}=[7.7 \%, 15 \%]\right)($ Fig. $6 \mathrm{~b})$. On the other hand, the Summary \& Random Choice model greatly underestimated the observed accuracy (predicted accuracy $=20 \% ; t(9)=-5.55$, $\left.p=3.55 \times 10^{-4}, \quad 95 \% \mathrm{CI}=[-13.5 \%,-5.7 \%]\right)$. Finally, the Summary \& Strategic Choice model produced the most accurate prediction (predicted accuracy $=33.3 \% ; t(9)=2.18, p=0.057$, $95 \% \mathrm{CI}=[-0.1 \%, 7.7 \%])$. On an individual subject level, the population model overestimated the accuracy of the second answer for all ten subjects, the Summary \& Random Choice model underestimated the accuracy of the second answer for all of the ten subjects, whereas the Summary \& Strategic Choice model was best calibrated overestimating the accuracy of the second answer for seven subjects and underestimating it for the remaining three subjects (Fig. 6c).

Formal comparisons of the model fits to the full distribution of responses for the second answers demonstrated that the population model provided the worst overall fit (Fig. 6d). Indeed, the population model resulted in AIC values that were higher than the Summary \& Random Choice model by an average of 18.05 points (corresponding to $8.29 \times 10^{3}$-fold difference in likelihood in the average subject) and a total of 180.46 points (corresponding to $1.53 \times 10^{39}$-fold difference in 
a
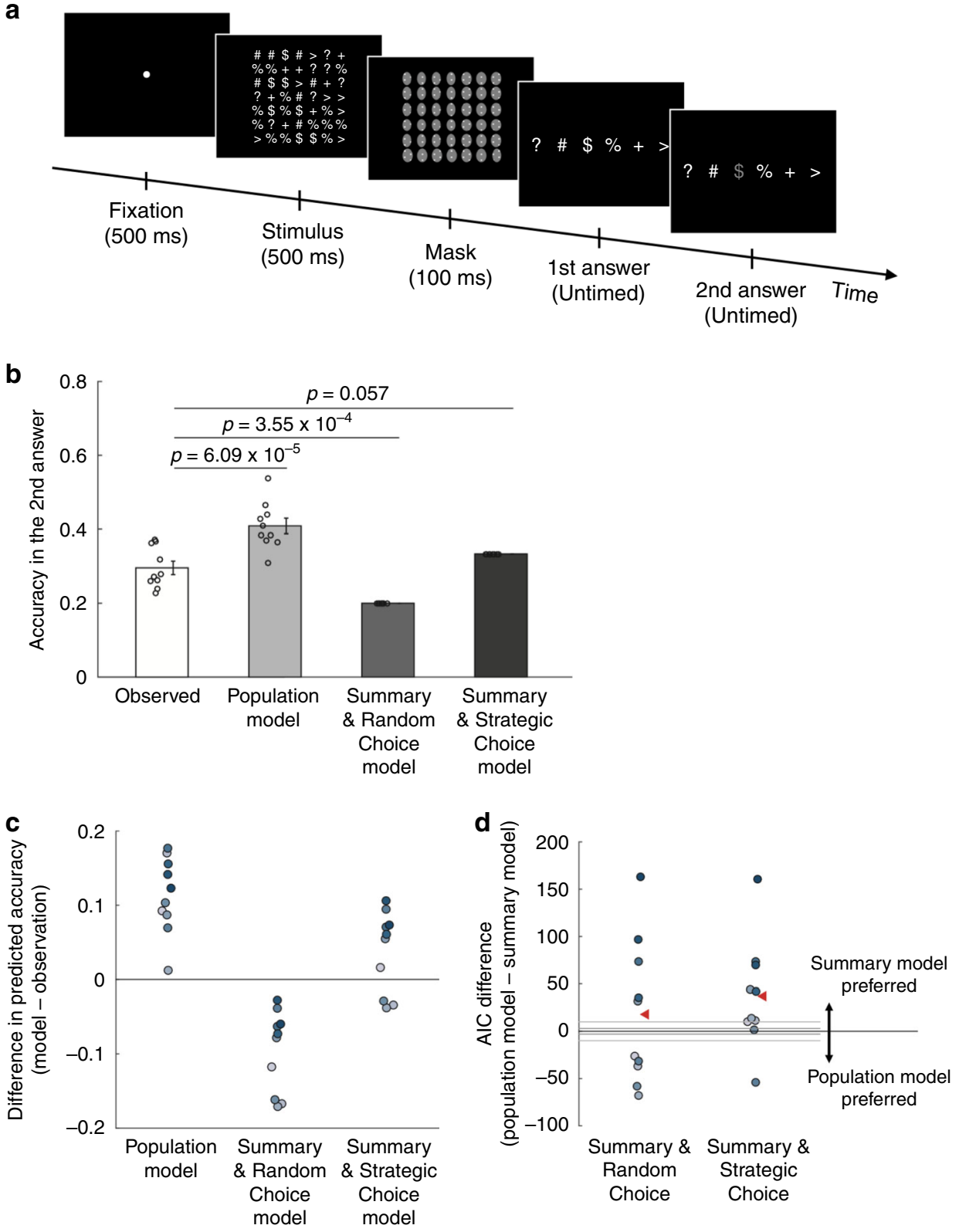

Fig. 6 Task and results for Experiment 3. a The same stimuli as in Experiment 2 were used in Experiment 3 but the task was slightly different. Subjects always reported the dominant symbol among all six alternatives. However, on $40 \%$ of the trials in which they gave a wrong answer, subjects were given the opportunity to make a second guess. b Mean accuracy for the second answer observed in the actual data (white bar), predicted by the population model (light gray bar), predicted by the Summary \& Random Choice model (dark gray bar), and predicted by the Summary \& Strategic Choice model (black bar). The predictions of the three models were derived based on subjects' first answers. All $p$ values are derived from two-sided paired $t$ tests. Error bars represent SEM, $n=10$. $\mathbf{c}$ Individual subjects' differences in the accuracy of the second answer between each model's prediction and the observed data. d AIC difference between the population and the two summary models. Positive AIC values indicate that the summary model provides a better fit to the data. Each dot represents one subject. The red triangle indicates the average AIC difference.

likelihood in the group). The population model underperformed the Summary \& Strategic Choice model even more severely (average AIC difference $=37.29$ points, corresponding to $1.25 \times 10^{8}$-fold difference in likelihood in the average subject; total AIC difference $=372.93$ points, corresponding to $9.57 \times$ $10^{80}$-fold difference in likelihood in the group). Lastly, the Summary \& Strategic Choice model also outperformed all of the additional models (Supplementary Results and Supplementary Fig. 6i-1). Thus, just as Experiments 1 and 2, Experiment 3 provides strong evidence that in the context of multi-alternative decisions, decision-making circuits only contain a summary of the sensory representation.
Experiment 4. To adjudicate between the population and summary models, Experiments 1-3 employed discrete stimulus categories (Fig. 1a). However, it remains possible that the results of these experiments would not generalize to stimuli represented on a continuous scale. To address this issue, we performed a fourth experiment that employed a feature (dot motion) represented on a continuous scale (degree orientation). We adapted the design of Treue et al. ${ }^{8}$ in which groups of dots slid transparently across one another. Specifically, we presented moving dot stimuli where three sets of dots moved in three different directions. As Treue et al. noted, these stimuli produce the subjective experience of seeing three distinct surfaces sliding across each other and 
a

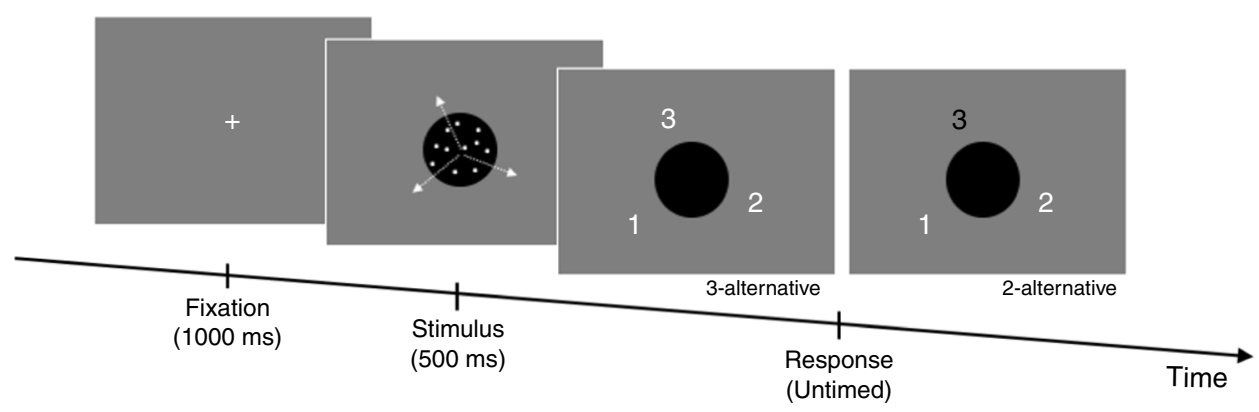

b

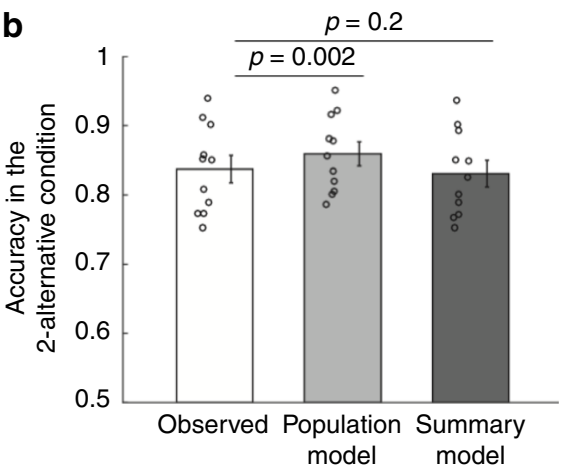

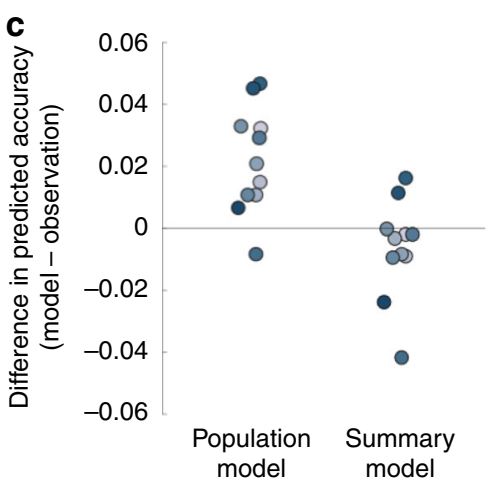

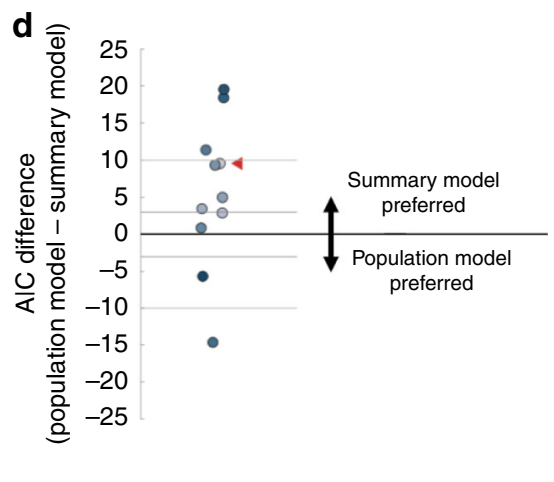

Fig. 7 Task and results for Experiment 4 which uses stimuli on a continuous scale. a Three sets of dots moved in three different directions separated by $120^{\circ}$. Similar to Experiments 1-3, one of the three sets of dots had more dots (i.e., dominant direction) compared to the other two sets (i.e., nondominant directions). Each trial began with a fixation cross followed by the moving dot stimulus presented for 500 ms. The response screen was presented

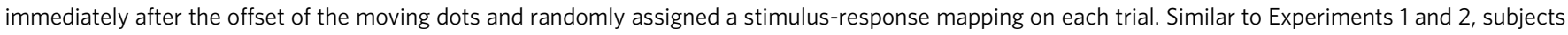

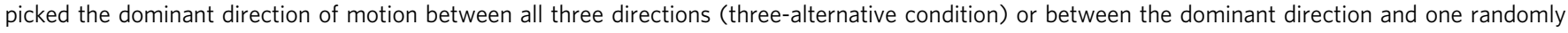

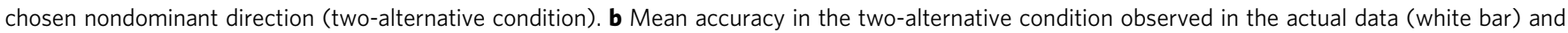
predicted by the population (light gray bar) and summary (dark gray bar) models. The predictions for both models were derived based on the data in the three-alternative condition. All $p$ values are derived from two-sided paired $t$ tests. Error bars represent SEM, $n=11$. c Individual subjects' differences in the accuracy of the two-alternative condition between the predictions of each of the two models and the observed data. $\mathbf{d}$ AIC difference between the

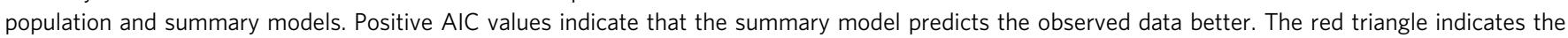
average AIC difference. The summary model fits better than the population model for nine out of the 11 subjects.

therefore give rise to a trimodal internal sensory distribution of motion direction. In each trial, one of the motion directions was represented by more dots (i.e., the dominant direction) than the other two (i.e., the nondominant directions). Subjects had to indicate the dominant direction of motion, which corresponds to the location of the tallest peak in the trimodal sensory distribution (Fig. 1b).

We used a similar modeling approach as in the previous three experiments (see "Methods") where we fitted a model of the sensory representation to the three-alternative condition (average accuracy $=77.4 \%$, chance level $=33.3 \%$ ), and compared the predicted accuracy of the two-alternative condition between the population and summary models. As in the previous experiments, we observed that the population model consistently overestimated the accuracy in the two-alternative condition (observed accuracy $=83.7 \%$; predicted accuracy $=$ $85.9 \%, t(10)=4.31, p=0.002,95 \%$ CI $[1.1 \%, 3.3 \%])$, whereas the summary model predicted the observed accuracy well (predicted accuracy $=83.1 \%, t(10)=-1.37, p=0.2,95 \% \mathrm{CI}=$ $[-1.7 \%, 0.4 \%]$ ) (Fig. $7 \mathrm{~b}$ ). In a direct comparison between the two models, the summary model predicted the observed task accuracy better for nine out of 11 subjects (Fig. 7c).

Finally, we compared the population and summary models' fits to the whole distribution of responses. On average, the AIC value of the summary model was lower by 5.47 points than the population model corresponding to the summary model being 15.40 times more likely for the average subject (Fig. $7 \mathrm{~d}$ ). The total
AIC difference across all subjects was 60.15 points lower for the summary model, corresponding to the summary model being $1.15 \times 10^{13}$ times more likely than the population model.

\section{Discussion}

We investigated whether the decision-level representation in decisions with multiple alternatives consists of a copy of the sensory representation or only a summary of it. We performed four experiments with either discrete stimulus categories or continuous stimuli producing multimodal distributions. The results across all experiments showed that the population model that assumes no loss of information from sensory to decisionmaking circuits did not provide a good fit to the data. Instead, the summary model, which assumes that decision-making circuits represent a reduced form of the sensory distribution, consistently provided a substantially better fit. These results strongly suggest that deliberate decision making for multiple alternatives only has access to a summary form of the sensory representation.

To the best of our knowledge, the current experiments are the first to address the question of whether complex sensory codes for a single stimulus can be accurately represented in decisionmaking circuits. Prior studies have convincingly demonstrated that humans can form complex, non-Gaussian ${ }^{9}$ and even bimo$\mathrm{dal}^{10}$ priors over repeated exposures to a given stimulus. However, it should be emphasized that this previous research has focused on the ability to learn a prior over many trials and did not 
examine the ability to use the sensory representation produced by a single stimulus on a single trial.

Why is it that decision-making circuits do not maintain a copy of the full sensory representation? While our study does not directly address the origins of this suboptimality, it is likely that the reason for the suboptimality lies in decision-making circuits having markedly smaller capacity than sensory circuits. One potential reason for the smaller capacity is that decision-making circuits have to be able to represent many different features (e.g., orientation, color, shape, object identity, etc.) each of which is processed in dedicated sensory regions. According to this line of reasoning, the generality inherent in decision-level representations necessitates that detail present in sensory cortex is lost. A related reason for the information loss in decision-making circuits is that the representations in such circuits often need to be maintained over a few seconds and therefore are subject to the well-known short-term memory decay ${ }^{11-14}$. For example, even though all conditions in our task were shown with a 0 -ms delay, information may need to be passed serially from sensory to decision-making circuits thus inherently inducing short-term memory demands. According to this line of reasoning, even if decision-making circuits can represent a full copy of the information in sensory cortex, that copy will decay as soon as it begins to be assembled and thus information loss with necessarily accrue before the representation can be used for subsequent computations. Therefore, both the necessary generality of decision-level representations and the inherent limitations of short-term memory likely contribute to the sparse representations in decision-making circuits.

If decision-making circuits indeed only have access to a summary form of the sensory representation, does that mean that absolutely no computations can be based on the complete sensory representations? There is evidence that decisions can take into account the full sensory representation both in simple situations where only two alternatives are present and in cases of automatic multisensory integration $3,15-19$. In general, automated computations performed directly on the sensory representations may use the whole sensory representation ${ }^{19}$. Therefore, certain types of decision making can be performed via mechanisms that do indeed take advantage of the entire sensory representation with either no or minimal loss of information. However, it is likely that such computations are restricted to either very simple decisions or processes that are already automated. On the other hand, the last stage of decision-making supporting nonautomatic, flexible, and deliberate decisions (which require short-term memory maintenance) only has access to a summary of the sensory representation.

Our findings can be misinterpreted as suggesting that complex visual displays are represented as a point estimate: that is, the decision-level representation features only the best guess of the system (e.g., $60^{\circ}$ orientation, red color, or the plus symbol). The possibility of a decision-level representation consisting of a single point estimate has been thoroughly debunked ${ }^{20-24}$. For example, a point estimate does not allow us to rate how confident we are in our decision because we lack a sense of how uncertain our point estimate is. Given that humans and animals can use confidence ratings to judge the likely accuracy of their decisions ${ }^{25-27}$, decision-making circuits must have access to more than a point estimate of the stimulus.

It should therefore be clarified that our summary model does not imply that decision making operates on point estimates. Indeed, as conceptualized in Fig. 1, the summary model assumes that subjects have access to both the identity of the most likely stimulus category (e.g., the color white) and the level of activity associated with that stimulus category. The level of activity can then be used as a measure of uncertainty, and confidence levels can be based on this level. Such confidence ratings will be less informative than the perceptual decision, which is exactly what has been observed in a number of studies ${ }^{2,28,29}$. In addition, this type of confidence generation may explain findings that confidence tends to be biased towards the level of the evidence for the chosen stimulus category and tends to ignore the level of evidence against the chosen category ${ }^{30-35}$. Thus, a summary model, consisting of the identity of the most likely stimulus and the level of activity associated with this stimulus, appears to be broadly consistent with findings related to how people compute uncertainty and is qualitatively different than a decision-level representation consisting only of a point estimate.

Another important question concerns whether any additional information is extracted from the sensory representation beyond what is assumed by the summary model. It is well known that humans can quickly and accurately extract the gist of a scene ${ }^{36-38}$, as well as the statistical structure of an image ${ }^{39}$. Therefore, it appears that rich information is extracted during the time when the stimulus is being viewed. In fact, this information often goes beyond the extraction of just the identity of the most likely stimulus and the level of activity associated with this stimulus assumed by our summary model. For example, our moving dots stimulus in Experiment 4 resulted in the perception of three surfaces sliding on top of each other. This means that what was extracted in that experiment was the approximate location of each of the three peaks of the trimodal sensory distribution. Similarly, the subjects in our Experiment 1 were certainly aware that four different colors were presented in each display and would have noticed if we ever presented additional colors. Subjects, therefore, had access to the identity of the different colors presented even though they did not have information about the activity level associated with each color. Thus, our summary model is likely to be an oversimplification of the actual representation used for decision-making. This point is further underscored by the fact that when predicting the accuracy in the two-alternative condition, the summary model showed a slight but systematic overprediction in Experiment 1 but underprediction in Experiment 2 (though it was better calibrated in Experiment 4).

Thus, we do not claim that rich information about the visual scene cannot be quickly and efficiently extracted (it can). What our results do suggest, however, is that decision-making circuits do not create a copy of the detailed sensory representation that can be used after the disappearance of the stimulus. This conclusion is reminiscent of the way deep convolutional neural networks (CNNs) operate: the decisions of these networks are based on compressed representations in the later layers rather than the detailed representations in the early layers ${ }^{40,41}$. In other words, even though CNNs extract complex representations in their early layers, the networks do not perform decision making based directly on these sensory-like representations in their early layers.

In conclusion, we found evidence from one exploratory (Experiment 1) and two preregistered (Experiments 2 and 3) studies that deliberate decision making for discrete stimulus categories is performed based on a summary of, rather than the whole, sensory representation. A final study (Experiment 4) extended these results to stimuli that give rise to continuous multimodal distributions. Our findings demonstrate that flexible computations may not be performed using the sensory activity itself but only a summary form of that activity.

\section{Methods}

Subjects. A total of 63 subjects participated in the four experiments. Experiment 1 had 32 subjects ( 15 females, mean age $=20.13, \mathrm{SD}=2.21$, range $=18-29$ ), Experiment 2 had 10 subjects $(7$ females, mean age $=20.5, \mathrm{SD}=3.06$, range $=$ $18-28$ ), Experiment 3 had 10 subjects ( 5 females, mean age $=20.8, \mathrm{SD}=3.55$, 
range $=18-28$ ), and Experiment 4 had 11 subjects ( 6 females, mean age $=21.45$, $\mathrm{SD}=2.5$, range $=20-28$ ). Each subject participated in only one experiment. All subjects provided informed consent and had normal or corrected-to-normal vision. The study was approved by the Georgia Tech Institutional Review Board (H15308).

Apparatus and experiment environment. The experiments stimuli were presented on a 21.5-inch iMac monitor in a dark room. The distance between the monitor and the subjects was $60 \mathrm{~cm}$. The stimuli were created in MATLAB, using Psychtoolbox $3^{42}$.

Experiment 1. The stimulus consisted of 49 circles colored in four different colors - red, blue, green, and white - presented in a $7 \times 7$ grid on black background. The diameter of each colored circle was $0.24^{\circ}$ and the distance between the centers of two adjacent circles was $0.6^{\circ}$. The grid was located at the center of the screen. On each trial, one of the four colors was dominant-it was featured in 16 different locations-whereas the other three colors were nondominant and were featured in 11 locations each. The exact locations of each color were pseudo-randomly chosen so that each color was presented the desired number of times.

A trial began with a $500-\mathrm{ms}$ fixation followed by $500-\mathrm{ms}$ stimulus presentation. Subjects then indicated the dominant color in the display and provided a confidence rating without time pressure.

There were three different conditions in the experiment. In the first condition, subjects could choose any of the four colors (four-alternative condition). In the second condition, after the stimulus offset subjects were asked to choose between only two options that were not announced in advance-one was always the correct dominant color and the other was a randomly selected nondominant color (twoalternative condition). Finally, in the third condition, subjects were told in advance which two colors will be queried at the end of the trial (advance warning condition). For the purposes of the current analyses, we only analyzed the fourand two-alternative conditions. The advanced warning condition and the confidence ratings were not analyzed.

Subjects completed six runs, each consisting of three 35-trial blocks (for a total of 630 trials). The three conditions used in the experiment were blocked such that one block in each run consisted entirely of trials from one condition and each run included one block from each condition. Subjects were given 15-s breaks between blocks and untimed breaks between runs. Before the start of the main experiment, subjects completed a training session where they completed 15 trials per condition with trial-to-trial feedback, and another 15 trials per condition without trial-to-trial feedback. No explicit feedback was provided during the main experiment in any of Experiments 1-4 though the presence of second answers in Experiment 3 served as a form of feedback that those specific trials were wrong. We did not hypothesize that the presence or absence of feedback would alter the results in a systematic way and therefore chose to withhold feedback as in our previous experiments ${ }^{43,44}$.

Experiment 2. Following our exploratory analyses on the data from Experiment 1, we preregistered two additional experiments (Experiments 2 and 3) ([osf.io/dr89k/]). These experiments were designed to generalize the results from Experiment 1 and to obtain stronger evidence for our model comparison results on the individual subject level. Consequently, we had fewer number of subjects in Experiments 2 and 3 but each subject completed many more trials. We ended up making three deviations from the preregistration: (1) we included a different number of locations for nondominant items (the preregistration wrongly indicated a number that is impossible given the number of categories and total number of characters), (2) we used a dot for the fixation even though the preregistration indicated that we would use a crosshairline, and (3) we tested additional models (the preregistration only included the population and summary models from Experiment 1).

The stimulus in Experiments 2 and 3 consisted of 49 characters from among six possible symbols (i.e.,?, \#, \$, \%, +, and $>$ ) presented in a $7 \times 7$ grid. The symbols were chosen to be maximally different from each other. The symbols' width was $0.382^{\circ}$ on average and height was $0.66^{\circ}$ on average. The distance between two centers of adjacent symbols was $1.1^{\circ}$. The symbols were presented in white on black background. On each trial, one of the six symbols was dominant-it was featured in 14 different locations-whereas the other five were nondominant and were featured in 7 locations each. The exact locations in the $7 \times 7$ grid where each symbol was displayed were pseudo-randomly chosen so that each symbol was presented the desired number of times.

Each trial began with a $500-\mathrm{ms}$ fixation, followed by a $500-\mathrm{ms}$ stimulus presentation. The stimuli were then masked for $100 \mathrm{~ms}$ with a $7 \times 7$ grid of ellipsoid-shaped images consisting of uniformly distributed noise pixels. Each ellipsoid had a width of $0.54^{\circ}$ and height of $0.95^{\circ}$, ensuring that it entirely covered each symbol. After the offset of the mask, subjects indicated the dominant symbol in the display without time pressure. No confidence ratings were obtained. The experiment had two conditions equivalent to the first two conditions in Experiment 1. In the first condition, subjects had to choose the dominant symbol among all six alternatives (six-alternative condition). In the second condition, subjects had to choose between two alternatives that were not announced in advance: the correct dominant symbol and a randomly selected nondominant symbol (two-alternative condition).
To obtain clear individual-level results, we collected data from each subject over the course of three different days. On each day, subjects completed 5 runs, each consisting of 4 blocks of 50 trials (for a total of 3000 trials per subject). We note that this very large number of trials makes it unlikely that any of our results in this or the subsequent experiments (which featured the same total number of trials) are due to insufficient training. The six- and two-alternative condition blocks were presented alternately, so that there were two blocks of each condition in a run. Subjects were given 15-s breaks between blocks and untimed breaks between runs. Before the start of the main experiment, subjects were given a short training on each day of the experiment.

Experiment 3. Experiment 3 used the same stimuli as in Experiment 2. Similar to Experiment 2, we presented a 500-ms fixation, a 500-ms stimulus, a 100-ms mask, and finally a response screen. Experiment 3 consisted of a single conditionsubjects always chose the dominant symbol among all six alternatives. However, on $40 \%$ of trials in which subjects gave a wrong answer, they were asked to provide a second answer by choosing among the remaining five symbols. Subjects could take as much time as they wanted for both responses. Subjects again completed 3000 trials over the course of three different days in a manner equivalent to Experiment 2.

Experiment 4. Experiment 4 employed a modified version of moving dots stimulus adapted from Treue et al. ${ }^{8}$. Three groups of dots moved in three different directions separated by $120^{\circ}$. Unlike most other experiments with moving dots, here all dots moved coherently in one of the three directions. The dots (density: $7.74 /$ degree $^{2}$; speed: $4^{\circ} / \mathrm{s}$ ) were white and were presented inside a black circle ( $3^{\circ}$ radius) positioned at the center of the screen on gray background. Each dot moved in one of the three directions and was redrawn to a random position if it went outside the black circle. In each trial, a dominant direction was randomly selected, and the two nondominant directions were fixed to $\pm 120^{\circ}$ from it. The dominant direction had a larger proportion of dots moving in that direction. This proportion was individually thresholded for each subject before the main experiment and was always greater than the proportions of dots moving in each nondominant direction (which were equal to each other).

Each trial began with a white fixation cross presented for $1 \mathrm{~s}$. The moving dots stimulus was then presented for $500 \mathrm{~ms}$, followed immediately by the response screen which randomly assigned the numbers 1,2 , and 3 to the three directions of motion (see Fig. 7a). Subjects' task was to press the keyboard number corresponding to the dominant motion direction. Similar to Experiments 1 and 2, there were two conditions: subjects chose the dominant direction of motion among all three directions (three-alternative condition) or between the dominant and one randomly chosen nondominant direction (two-alternative condition). In the twoalternative condition, the two available options were colored in white and the unavailable option was colored in black.

Each subject completed three sessions of the experiment on different days. Each session started with a short training session. On the first day, subjects completed six blocks of 40 trials with the three-alternative condition. The proportion of dots moving in the dominant direction was initially set to $60 \%$. After each block, we updated the proportion of dots moving in the dominant direction such that the proportion of dots for the dominant direction increased by $10 \%$ if accuracy was lower than $60 \%$ or decreased by $10 \%$ if accuracy was greater than $80 \%$. Once the task accuracy fell in the $60-80 \%$ range, the proportion of dots moving in the dominant direction was adjusted by half of a previous proportion change. After the six blocks, subjects' performance was reviewed by an experimenter who could further adjust the proportion of dots moving in the dominant direction. Once selected, the proportion of dots moving in the dominant direction was fixed for al sessions. Each session had 5 runs, each consisting of 4 blocks of 50 trials (for a total of 3000 trials per subject). The three- and two-alternative conditions were presented in alternate blocks with the condition presented first counterbalanced between subjects.

Model development for Experiments 1-3. We developed and compared two main models of the decision-level representation. According to the population model, decision-making circuits have access to the whole sensory representation. On the other hand, according to the summary model, decision-making circuits only have the access to a summary of the sensory representation but not to the whole sensory distribution.

In order to compare the population and summary models, we first had to develop a model of the sensory representation. We created this model using the four- and six-alternative conditions in Experiments 1 and 2, and the first answer in Experiment 3 . The population and summary models were then used to make predictions about the two-alternative condition in Experiments 1 and 2, and the second answer in Experiment 3. These predictions were made without the use of any extra parameters.

We created a model of the sensory representation for Experiment 1 as follows. We assumed that each of the four types of stimuli (red, blue, green, or white being the dominant color) produced variable across-trial activity corresponding to each of the four colors. We modeled this activity as Gaussian distributions whose mean $(\mu)$ is a free parameter and variance is set to one. However, in our experiments, the 
perceptual decisions only depended on the relative values of the activity levels and not on their absolute values. In other words, adding a constant to all four $\mu$ s for a given dominant stimulus would result in equivalent decisions. Therefore, without loss of generality, we set the mean for the activity corresponding to each dominant color as 0 . This procedure resulted in 12 different free parameters such that for each of the 4 possible dominant colors there were $3 \mu$ s corresponding to each of the nondominant colors. Finally, we included an additional parameter that models the lapse rate. Note that the inclusion of a lapse rate parameter has a greater influence on percent correct in the two-alternative compared to the four-alternative condition because overall performance is higher in the two-alternative condition. Therefore, introducing a lapse rate parameter favors the population model by leading to predictions of lower performance in the two-alternative condition (which helps the population model since it consistently predicts higher performance than what was empirically observed).

The sensory representation was modeled in a similar fashion in Experiments 2 and 3. In both cases, the model was created based on subjects choosing between all available options (i.e., the six-alternative condition in Experiment 2 and the first answer in Experiment 3). The model of the sensory representation in Experiments 2 and 3 thus had 30 free parameters related to the sensory activations (for each of the six possible dominant symbols there were five $\mu$ s corresponding to each of the nondominant symbols) and an additional free parameter for the lapse rate.

We modeled the activations produced by each stimulus type separately to capture potential relationships between different colors or symbols (e.g., some color pairs may be perceptually more similar than others). However, we re-did all analyses using the simplifying assumption that when a color is nondominant, that color has the same $\mu$ regardless of which the dominant color is. This assumption allowed us to significantly reduce the number of parameters in our model of the sensory representation. In this alternative model of the sensory representation, the mean activity for each color/symbol was determined only based on whether that color/symbol was dominant or not. Therefore, we included two free parameters for each color/symbol. However, because of the issue described above (adding a constant to all $\mu \mathrm{s}$ in a given experiment would result in identical decisions), we fixed one of the $\mu$ s to 0 . This modeling approach reduced the total number of free parameters to eight in Experiment 1 (seven $\mu$ s and a lapse rate) and 12 free parameters in Experiments 2 and 3 (11 $\mu$ s and a lapse rate). This modeling approach produced virtually the same results (Supplementary Fig. 5).

Lastly, we considered two different instantiations of the summary model for Experiment 3. In the first instantiation, which we refer to as the Summary \& Random Choice model, it is assumed that when the first answer is wrong, then the subject would randomly pick a second answer among the remaining options. In the second instantiation, which we refer to as the Summary \& Strategic Choice model, it is assumed that when the first answer is wrong, then the subject would pick the stimulus category of a randomly recalled symbol from the original $7 \times 7$ grid that is different from the stimulus category chosen with the first answer. According to this model, the subject would pick the second answer correctly $33.3 \%$ of the time and each incorrect symbol will be chosen $16.7 \%$ of the time (Supplementary Fig. 4).

Model development for Experiment 4. Experiment 4 employed moving dots and required subjects to indicate the dominant direction of motion. Therefore, unlike the tasks in Experiments 1-3 that were based on discrete categories of stimuli, the task in Experiment 4 featured a continuous variable (direction of motion, varying from $0^{\circ}$ to $360^{\circ}$ ). However, despite the continuous nature of the stimulus, the three directions of motion could be easily identified implying that the stimulus resulted in a trimodal sensory distribution ${ }^{8}$. This allowed us to use the same modeling approach from Experiments 1-3 by essentially treating the three motion directions as discrete stimuli. We again developed a model of the sensory representation that was fit to the three-alternative condition. Unlike Experiments 1-3 where the categories of stimuli were fixed, here the dominant direction of motion was chosen randomly (from $0^{\circ}$ to $360^{\circ}$ ) on every trial. Therefore, the model only had parameters for the heights of the nondominant and dominant directions of motion. Because, just as in the previous experiments, adding a constant to all both parameters would result in identical decisions, the parameters for the nondominant direction was fixed thus leaving us with a single free parameter. Once the model was fit to the data from the three-alternative condition, the population and summary models had no free parameters when applied to the data from the twoalternative condition.

Model fitting and model comparison. For all four experiments, we fit the models to the data as previously $y^{45-48}$ using a maximum likelihood estimation approach. The models were fit to the full distribution of probabilities of each response type contingent on each stimulus type:

$$
\text { Log likelihood }=\sum_{i, j} \log \left(p_{i j}\right) \times n_{i j},
$$

where $p_{i j}$ is the predicted probability of giving a response $i$ when stimulus $j$ is presented, whereas $n_{i j}$ is the observed number of trials where a response $i$ was given when stimulus $j$ was presented. We give formulas for computing $p_{i j}$ in a simplified model without a lapse rate in the Supplementary Methods. Because the analytical expressions to obtain $p_{i j}$ are difficult to compute, we derived the model behavior for every set of parameters by numerically simulating 100,000 individual trials with that parameter set. Model fitting was done by finding the maximum-likelihood parameter values using simulated annealing ${ }^{49}$. Fitting was conducted separately for each subject.

Based on the parameters of the model describing the sensory representation, we generated predictions for the two-alternative condition (Experiments 1,2, and 4) and the second answer (Experiment 3 ) for both the population and summary models. These predictions contained no free parameters. To compare the models, we computed AIC based on the $\log$-likelihood $(\log (\mathcal{L}))$ for each model using the formula:

$$
\mathrm{AIC}=-2 \times \log (\mathcal{L})+2 \times k,
$$

where $k$ is the number of parameters of a model. Because both the population and summary models had no free parameters, this formula simplifies to $\mathrm{AIC}=-2 \times \log (\mathcal{L})$. Note other measures, such as the AIC corrected for small sample sizes (AICc) given by the formula:

$$
\mathrm{AICc}=-2 \times \log (\mathcal{L})+2 \times k+\frac{2\left(k^{2}+k\right)}{n-k-1},
$$

where $n$ is the total number of observations, and the Bayesian Information Criterion (BIC) given by the formula:

$$
\mathrm{BIC}=-2 \times \log (\mathcal{L})+k \times \log (n)
$$

would result in the exact same values as AIC for $k=0$. We chose to report AIC values instead of the raw $\log (\mathcal{L})$ values because of their wider usage and larger familiarity but all conclusions would remain the same if the $\operatorname{raw} \log (\mathcal{L})$ values are considered. Note that lower AIC values correspond to better model fits.

Statistical tests. All statistical tests reported are two-sided paired $t$ tests.

Reporting summary. Further information on research design is available in the Nature Research Reporting Summary linked to this article.

\section{Data availability}

Model fitting data and results of the four experiments are available at the OSF repository (https://osf.io/d2b9v) ${ }^{50}$. In addition, the data from Experiment 1 were made available on the Confidence Database (https://osf.io/s46pr//) ${ }^{51}$. A reporting summary for this article is available as a Supplementary Information file. Source data are provided with this paper.

\section{Code availability}

The analysis codes for all four experiments are available at the OSF repository (https:// osf.io/d2b9v) $)^{50}$.

Received: 28 February 2019; Accepted: 8 July 2020; Published online: 31 July 2020

\section{References}

1. von Helmholtz, H. Treatise on Physiological Optics (Thoemmes Continuum, 1856).

2. Rahnev, D. \& Denison, R. N. Suboptimality in perceptual decision making. Behav. Brain Sci. 41, e223 (2018).

3. Churchland, A. K., Kiani, R. \& Shadlen, M. N. Decision-making with multiple alternatives. Nat. Neurosci. 11, 693-702 (2008).

4. Drugowitsch, J., Wyart, V., Devauchelle, A. D. \& Koechlin, E. Computational precision of mental inference as critical source of human choice suboptimality. Neuron 92, 1398-1411 (2016).

5. Forstmann, B. U., Ratcliff, R. \& Wagenmakers, E.-J. Sequential sampling models in cognitive neuroscience: advantages, applications, and extensions. Annu. Rev. Psychol. 67, 641-666 (2016).

6. Green, D. M. \& Swets, J. A. Signal Detection Theory and Psychophysics (John Wiley \& Sons Ltd., 1966).

7. Ratcliff, R. A theory of memory retrieval. Psychol. Rev. 85, 59-108 (1978).

8. Treue, S., Hol, K. \& Rauber, H.-J. Seeing multiple directions of motionphysiology and psychophysics. Nat. Neurosci. 3, 270-276 (2000).

9. Knill, D. C. Learning Bayesian priors for depth perception. J. Vis. 7, 13 (2007).

10. Körding, K. P. \& Wolpert, D. M. Bayesian integration in sensorimotor learning. Nature 427, 244-247 (2004).

11. Sims, C. R., Jacobs, R. A. \& Knill, D. C. An ideal observer analysis of visual working memory. Psychol. Rev. 119, 807-830 (2012).

12. Craik, F. I. M. \& Jennings, J. M. in The Handbook of Aging and Cognition (eds Craiks, F. I. M. \& Salthouse, T. A.) 51-110 (Lawrence Erlbaum Associates, Inc., 1992).

13. MacGregor, J. N. Short-term memory capacity: limitation or optimization? Psychol. Rev. 94, 107-108 (1987). 
14. Cowan, N. Metatheory of storage capacity limits. Behav. Brain Sci. 24, 154-176 (2001).

15. Prsa, M., Gale, S. \& Blanke, O. Self-motion leads to mandatory cue fusion across sensory modalities. J. Neurophysiol. 108, 2282-2291 (2012).

16. Saarela, T. P. \& Landy, M. S. Integration trumps selection in object recognition. Curr. Biol. 25, 920-927 (2015).

17. Hillis, J. M. Combining sensory information: mandatory fusion within, but not between, senses. Science 298, 1627-1630 (2002).

18. Beck, J. M. et al. Probabilistic population codes for Bayesian decision making. Neuron 60, 1142-1152 (2008).

19. Hou, H., Zheng, Q., Zhao, Y., Pouget, A. \& Gu, Y. Neural correlates of optimal multisensory decision making under time-varying reliabilities with an invariant linear probabilistic population code. Neuron 104, 1010-1021.e10 (2019).

20. Ma, W. J. Signal detection theory, uncertainty, and poisson-like population codes. Vis. Res. 50, 2308-2319 (2010).

21. Ma, W. J. \& Jazayeri, M. Neural coding of uncertainty and probability. Annu. Rev. Neurosci. 37, 205-220 (2014).

22. Knill, D. C. \& Pouget, A. The Bayesian brain: the role of uncertainty in neural coding and computation. Trends Neurosci. 27, 712-719 (2004).

23. Fiser, J., Berkes, P., Orbán, G. \& Lengyel, M. Statistically optimal perception and learning: from behavior to neural representations. Trends Cogn. Sci. 14, 119-130 (2010).

24. Pouget, A., Dayan, P. \& Zemel, R. S. Inference and computation with population codes. Annu. Rev. Neurosci. 26, 381-410 (2003).

25. Metcalfe, J. \& Shimamura, A. P. Metacognition: Knowing About Knowing (The MIT Press, 1996).

26. Mamassian, P. Visual confidence. Annu. Rev. Vis. Sci. 2, 459-481 (2016).

27. Kiani, R. \& Shadlen, M. N. Representation of confidence associated with a decision by neurons in the parietal cortex. Science 324, 759-764 (2009).

28. Maniscalco, B. \& Lau, H. A signal detection theoretic approach for estimating metacognitive sensitivity from confidence ratings. Conscious. Cogn. 21, 422-430 (2012).

29. Rahnev, D., Koizumi, A., McCurdy, L. Y., D’Esposito, M. \& Lau, H. Confidence leak in perceptual decision making. Psychol. Sci. 26, 1664-1680 (2015).

30. Peters, M. A. K. et al. Perceptual confidence neglects decision-incongruent evidence in the brain. Nat. Hum. Behav. 1, 1-8 (2017).

31. Koizumi, A., Maniscalco, B. \& Lau, H. Does perceptual confidence facilitate cognitive control? Atten., Percept., Psychophys. 77, 1295-1306 (2015).

32. Zylberberg, A., Barttfeld, P. \& Sigman, M. The construction of confidence in a perceptual decision. Front. Integr. Neurosci. 6, 79 (2012).

33. Maniscalco, B., Peters, M. A. K. \& Lau, H. Heuristic use of perceptual evidence leads to dissociation between performance and metacognitive sensitivity. Atten., Percept., Psychophys. 78, 923-937 (2016).

34. Samaha, J., Barrett, J. J., Sheldon, A. D., LaRocque, J. J. \& Postle, B. R. Dissociating perceptual confidence from discrimination accuracy reveals no influence of metacognitive awareness on working memory. Front. Psychol. 7, 851 (2016)

35. Talluri, B. C., Urai, A. E., Tsetsos, K., Usher, M. \& Donner, T. H. Confirmation bias through selective overweighting of choice-consistent evidence. Curr. Biol. 28, 3128-3135.e8 (2018).

36. Greene, M. R. \& Oliva, A. The briefest of glances: the time course of natural scene understanding. Psychol. Sci. 20, 464-472 (2009).

37. Potter, M. C. Short-term conceptual memory for pictures. J. Exp. Psychol Hum. Learn. Mem. 2, 509-522 (1976).

38. Potter, M. C. Meaning in visual search. Science 187, 965-966 (1975).

39. Fiser, J. \& Aslin, R. N. Unsupervised statistical learning of higher-order spatial structures from visual scenes. Psychol. Sci. 12, 499-504 (2001).

40. Kriegeskorte, N. Deep neural networks: a new framework for modeling biological vision and brain information processing. Annu. Rev. Vis. Sci. 1, 417-446 (2015).

41. Kubilius, J., Bracci, S. \& Op de Beeck, H. P. Deep neural networks as a computational model for human shape sensitivity. PLOS Comput. Biol. 12, e1004896 (2016).

42. Pelli, D. G. The VideoToolbox software for visual psychophysics: Transforming numbers into movies. Spat. Vis. 10, 437-42 (1997).
43. Yeon, J., Shekhar, M. \& Rahnev, D. Overlapping and unique neural circuits support perceptual decision making and confidence. Preprint at https://doi. org/10.1101/439463v2 (2020).

44. Shekhar, M. \& Rahnev, D. Distinguishing the roles of dorsolateral and anterior PFC in visual metacognition. J. Neurosci. 38, 5078-5087 (2018).

45. Bang, J. W., Shekhar, M. \& Rahnev, D. Sensory noise increases metacognitive efficiency. J. Exp. Psychol. Gen. 148, 437-452 (2019).

46. Rahnev, D. et al. Attention induces conservative subjective biases in visual perception. Nat. Neurosci. 14, 1513-1515 (2011).

47. Rahnev, D. et al. Continuous theta burst transcranial magnetic stimulation reduces resting state connectivity between visual areas. J. Neurophysiol. 110 1811-1821 (2013).

48. Rahnev, D., Maniscalco, B., Luber, B., Lau, H. \& Lisanby, S. H. Direct injection of noise to the visual cortex decreases accuracy but increases decision confidence. J. Neurophysiol. 107, 1556-1563 (2012).

49. Kirkpatrick, S., Gelatt, C. D. \& Vecchi, M. P. Optimization by simulated annealing. Science 220, 671-680 (1983).

50. Yeon, J. \& Rahnev, D. On the nature of the perceptual representation at the decision stage. OSF https://doi.org/10.17605/OSF.IO/D2B9V (2020).

51. Rahnev, D. et al. The Confidence Database. Nat. Hum. Behav. https://doi.org/ 10.1038/s41562-019-0813-1 (2020).

\section{Acknowledgements}

We thank Tristan Hackman, Megan Kelley, and Milan Patel for help with the data collection. This work was supported by the National Institute of Mental Health of the National Institutes of Health under Award Number R01MH119189 to D.R.

\section{Author contributions}

J.Y. and D.R. designed the study. J.Y. collected and analyzed data. Both J.Y. and D.R. wrote the manuscript and approved the final version for submission.

\section{Competing interests}

The authors declare no competing interests.

\section{Additional information}

Supplementary information is available for this paper at https://doi.org/10.1038/s41467 020-17661-z.

Correspondence and requests for materials should be addressed to J.Y. or D.R.

Peer review information Nature Communications thanks Michael Landy, and the other, anonymous, reviewer(s) for their contribution to the peer review of this work

Reprints and permission information is available at http://www.nature.com/reprints

Publisher's note Springer Nature remains neutral with regard to jurisdictional claims in published maps and institutional affiliations.

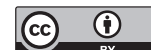

Open Access This article is licensed under a Creative Common Attribution 4.0 International License, which permits use, sharing, adaptation, distribution and reproduction in any medium or format, as long as you give appropriate credit to the original author(s) and the source, provide a link to the Creative Commons license, and indicate if changes were made. The images or other third party material in this article are included in the article's Creative Commons license, unless indicated otherwise in a credit line to the material. If material is not included in the article's Creative Commons license and your intended use is not permitted by statutory regulation or exceeds the permitted use, you will need to obtain permission directly from the copyright holder. To view a copy of this license, visit http://creativecommons.org/ licenses/by/4.0/

(c) The Author(s) 2020 